\title{
LA PRÉSENCE DES GERMES PEPTONISANTS ET THERIVO-RÉSISTANTS DANS LES LAITS
}

\author{
par Mare FOUASSIER. \\ de l'Institut Pasteur.
}

Dans un article fort intéressant paru dans cette Revue (1) M. Lind, entre autres moyens pour apprécier le degré de chauffage qu'un lait aurait subi, fait intervenir l'épreuve de la catalase, celle-ci devant mettre en évidence la persistance plus ou moins grande des enzymes dans ce lait, en rapport avec son degré de chauffage, et la durée de celui-ci.

Le paragraphe qui traite de cette question a d'autant plus retenu mon attention que voici plusieurs années, je l'avais étudiée, sans doute par une technique et dans un but différents de ceux de M. LIND, mais qui cependant m'avaient permis de tirer des conclusions intéressantes pour l'industrie laitière.

Dans la pratique, la catalasimétrie ne peut donner que des résultats très imparfaits dans leur précision et leur interprétation. Si les enzymes naturelles du lait sont bien progressivement détruites au fur et à mesure de l'élévation de température, comme le confirme M. LiND, il en est d'autres d'origine microbienne, qui agissent plus ou moins violemment sur l'eau oxygénée, et qui interviennent d'une manière tout à fait imprévue pour bouleverser ces résultats.

De nombreux auteurs, parmi lesquels il faut citer KonING, SARTHOU, O. JENSEN avaient d'ailleurs, bien antérieurement à mes constatations, fait observer, dans le cas des laits crus, la superposition des actions de la catalase naturelle et de la catalase microbienne dans la décomposition de l'eau oxygénée.

Il m'avait paru alors intéressant d'essayer d'appliquer la catalasimétrie aux laits normalement pasteurisés, c'est-à-dire à céux dans lesquels la catalase physiologique aurait été détruite, donc éliminée, dans l'appréciation des résultats, a fin de déterminer le degré de pollution microbienne d'un lait post-pasteurisation abandonné au contact de l'air dans les conditions normales de la pratique laitière, ou encore subissant l'action des germes thermo-résistants momentanément suspendue.

Voici comment j'exposais en deux notes la technique suivie et les résultats obtenus :

$1^{0}$ Uń certain nombre de laits pasteurisés, de consommation journalière ont été ensemencés sur gélose lactosée. Outre les ferments lactiques, j'ai isolé plusieurs variétés de germes, pármi lesquels le subtilis, le tyrothrix tenuis, l'oüdium lactis et une levure de lactose se sont

(1) C. LrNd. Les enzymes du lait et leur utilisation pour apprécier le chauffage d'un jait. Le Lait 1927, No 70 , p. 937 ot suiv. 
rencontrés le plus fréquemmént. Afin de déterminer comparativement leur action vis-à-vis l'eau oxygénée, ces germes, à l'état de pureté, ont été ensuite ensemencés sur un milieu lactosé peptoné et stérilisé contenu dans une série de tubes à essai. Un lot est conservé comme témoin, un autre reçoit 1 pour 100 d'eau oxýgénée à 12 vol. un autre enfin 4 p. 100 d'eau oxygénée, puis le tout est porté à l'étuve à $30^{\circ}$.

J'indique dans le tableau suivant les résultats enregistrés après 48 heures d'incubation, terme qui m'a semblé le plus favorable pour faire mieux ressortir les différences qui existent entre les divers essais.

\begin{tabular}{|c|c|c|c|c|c|c|}
\hline & Témoin. & $\begin{array}{l}\text { Pouvoir } \\
\text { catalysant } \\
\text { du témoin. }\end{array}$ & $\begin{array}{l}\text { Essai } \\
1 \% \\
\mathrm{H}^{2} \mathrm{O}^{2} .\end{array}$ & $\begin{array}{c}\text { Recherche } \\
\text { de } \\
\mathrm{H}^{2} \mathrm{O}^{2} \text {. }\end{array}$ & $\begin{array}{l}\text { Essai } \\
4 \% \\
\mathrm{H}^{2} \mathrm{O}^{2} \\
-\end{array}$ & $\begin{array}{c}\text { Recherche } \\
\text { de } \\
\mathrm{H}^{2} \mathrm{O}^{2} .\end{array}$ \\
\hline Subtilis .......... & ++ & 9,1 & ++ & absence & ++ & absence \\
\hline Tyrothrix ....... & ++ & 8,4 & ++ & $n$ & ++ & 》 \\
\hline Oïdium .... . & ++ & 1,5 & + & 》) & 0 & présence \\
\hline Levure.......... & ++ & 0,6 & + & ” & 0 & 》 \\
\hline Ferment lactique & ++ & 0. & 0 & présence & 0 & 》 \\
\hline
\end{tabular}

Le signe + indique une culture moyenne, le signe ++ une culture abondante et le signe 0 une culture nulle.

Le pouvoir catalysant a été déterminé en mélangeant intimement dans un uromètre $10 \mathrm{~cm}^{3}$ du milieu liquide témoin ayant donné une culture abondante avec $5 \mathrm{~cm}^{3} \mathrm{~d}^{\prime} e a u$ oxygénée à $12 \mathrm{vol}$. Le volume d'oxygène dégagé $a$ été mesuré après 30 minutes de contact.

On remarque que, pour tous les tubes dans lesquels les germes se sont développés, la recherche pour retrouver l'eau oxygénée n'a pas eu de résultats, alors qu'elle a donné un résultat positif pour les tubes où le développement microbien ne s'est pas produit.

Le subtilis et le tyrothrix possèdant un pouvoir catalysant élevé ont rapidement décomposé l'eau oxygénée, ajoutée même à des doses massives et se sont développés, dans ce cas, avec la même intensité que dans le témoin.

Les autres germes ont un pouvoir catalysant de plus en plus faible qui tombe à zéro pour le ferment lactique dans les conditions où je me suis placé : la dose la plus faible d'eau oxygénée a donc suffi à empêcher le développement de ce ferment.

Je me suis assuré que la décomposition de l'eau oxygénée constatée dans mes essais était imputable à une action diastasique. Cette action continue, en effet, à se manifester après filtration du milieu de culture sur bougie, tandis qu'elle est arrêtée par chauffage de ce milieu à $80^{\circ}$.

En terminant, je ferai observer que les germes pour lesquels le pouvoir catalysant est le plus élevé sont pourvus de spores qui peuvent résister à la pasteurisation.

$2^{\circ}$ Dans une précédente note, j'ai exposé que certains germes $(B . s u b$ - 
tilis, tyrothrix tenuis), fréquemment rencontrés dans le lait pasteurisé, possédaient la propriété de décomposer activement l'eau oxygénée, en proportions variables, lorsqu'ils étaient cultivés sur un milieu approprié.

J'ai étudié si cette action continuait à se manifester dans le lait, en outre, j'ai déterminé l'influence que ces germes exerçaient dans les mêmes eonditions, sur la fermentation lactique en présence ou en l'absence du bioxyde d'hydrogène.

Pour cela, une série de tubes à essais contenant une quantité égale de lait stérilisé, les uns sans aucune addition, les autres additionnés de $1 \%$ d'eau oxygénée à 12 vol. sont ensemencés respectivement avec le B. subtilis, le Tyrothrix ou le ferment lactique d'une part, et avec le mélange $B$. subtilis-ferment lactique ou Tyrothrix-ferment lactique d'autre part.

Les ensemencements étaient effectués à l'aide de quelques gouttes d'une émulsion aqueuse de culture microbienne.

Après 15 heures d'incubation à $30^{\circ}$, j'ai déterminéle pouvoir catalysant sur une série de ces tubes et l'acidité lactique sur l'autre série.

Les résultats obtenus sont consignés dans le tableau suivant.

\section{HEURES APRÈS L'ENSEMENCEMENT.}

\begin{tabular}{|c|c|c|c|c|}
\hline \multirow[b]{2}{*}{$\begin{array}{l}\text { Culture } \\
\text { sur lait stérilisé. } \\
\text { de }\end{array}$} & \multirow{2}{*}{$\begin{array}{c}\text { Décomposition } \\
\text { de } \text { l'H }^{2} \mathrm{O}^{2} \text { ajoutée } \\
\text { préalablement } \\
\text { à la dose } \\
\text { de } 1 \text { p. } 100\end{array}$} & \multirow[b]{2}{*}{$\begin{array}{c}\text { Pouvoir } \\
\text { catalysant } \\
\text { de } \\
\text { cultures }\end{array}$} & \multicolumn{2}{|c|}{$\begin{array}{l}\text { Aciditité lactique } \\
\text { de la culture exprimée } \\
\text { en degrès Dornic. }\end{array}$} \\
\hline & & & $\begin{array}{l}\text { Addition } \\
\text { de } 1 \mathrm{p} .100 \\
\mathrm{H}^{2} \mathrm{O}^{2}\end{array}$ & $\begin{array}{c}\text { Sans } \\
\text { addition }\end{array}$ \\
\hline & totale & 5,8 & $19^{\circ} 8$ & $19^{\circ} 8$ \\
\hline & ” & 2,2 & $20^{\circ} 2$ & $20^{\circ} 1$ \\
\hline tique ......... & " & 0,2 & $27^{\circ} 0$ & 5202 \\
\hline+ ferment lactiqu & $\Rightarrow$ & 6,1 & $74^{\circ} 7$ & $77^{\circ} 4$ \\
\hline+ ferment lactiq & ” & 2,3 & $72^{\circ} 0$ & $76^{\circ} 5$ \\
\hline témoin. & nulle & nul & $19^{\circ} 8$ & $19 \circ 8$ \\
\hline
\end{tabular}

En ce qui concerne le pouvoir catalysant, on remarque que mes constatations antérieures, sur milieu lactosé, se trouvent confirmées pour le $B$. subtilis et le Tyrothrix, tandis que le pouvoir catalysant du ferment lactique, précédemment nul dans ce même milieu, est maintenant suffisamment accusé pour permettre la décomposition totale de l'eau oxygénée à la dose de $1 \%$. Quant aux' associations microbiennes, on constate que les pouvoirs catalysants des germes en présence se sont additionnés, l'eau oxygénée a été de ce fait rapidement détruite.

En considérant les chiffres d'acidité, on voit que le $B$. subtilis et le Tyrothrix donnent sensiblement le même chiffre que le lait stérilisé témoin. L'action acidi fiante du ferment lactique a été totalement arrêtée, 
ainsi que je m'en suis assuré par les déterminations, à intervalle régulier, tantque son action catalysante n'avait pas entièrement décomposé $\mathbf{H}^{2} \mathrm{O}^{2}$.

Dans le cas des associations microbiennes, non seulement les chiffres d'acidité sont très voisins les uns des autres, ce qui démontre combien le rôle antiseptique de $\mathrm{I}^{\prime} \mathrm{H}^{2} \mathrm{O}^{2}$ a été de peu de durée, mais encore ces chiffres accusent une acidification beaucoup plus considérable que lorsque le ferment lactique intervient seul dans les mêmes conditions. Cette aetion avait du reste été déjà observée quant aux Tyrothrix.

On peut conclure que l'eau oxygénée, ajoutée après pasteurisation aura une action antiseptique vis-à-vis les ferments lactiques que le lait collecte pendant le temps variable qui précède sa consommation. Cette action sera d'autant plus prolongée que le lait aura été tenu à l'abri des poussières chargées de $B$. subtilis ou de Tyrothrix, eomme celles des étables par exemple. Les spores de ces germes résistant à la pasteurisation, ceux-ci pourront, par la suite, proliférer dans le lait. Par leurs propriétés catalysantes, ils contribueront pour une large part à la décomposition totale de l'eau oxygénée, au cas où cet antiseptique aurait été employé; en outre, ils activeront le développement des ferments lactiques en fournissant à ceux-ci un élément plus facilement assimilable, grâce à l'action peptonisante qu'ils exercent sur les albuminoïdes du lait.

$$
*^{*} *
$$

Dans un lait pasteurisé, il se reforme donc une catalase d'origine microbienne par simple apport de germes extérieurs, d'une part, et par la reprise de vitalité des germes sporulés ayant résisté à la pasteurisation, d'autre part. En l'absence de ces germes, un lait pasteurisé avec soin conservé à l'abri de l'air devrait conserver un pouvoir catalysant nul pendant le temps normal de vente à la consommation. M. LiNd va même plus loin en disant que la catalase ne réapparaîtra pas.

S'il est logique d'entrevoir le rôle de l'air dans la contamination du lait pasteurisé, il ne faut pas davantage perdre de vue que le lait pasteurisén'est pas du lait stérilisé. Le premier qui fit cette constatation n'estce pas PASTEUR lui-même? Que l'on me permette la citation suivante : "Le 10 avril 1860, écrit-il, je prépare un ballon de lait. L'ébullition a duré deux minutes, depuis le moment où la vapeur d'eau avait déjà assez échauffé la partie effilée du col pour que l'on ne puisse y tenir la main. Après refroidissement du liquide, on ferme à la lampe le col du ballon comme à l'ordinaire, et on le porte dans une étuve à la température de 25 à $30^{\circ}$. Le 17 avril, le lait de ce ballon était caillé, »

Quand PASTEUR parle d'une fermeture comme à l'ordinaire, cela indique que l'air rentré dans le ballon à la suite de son refroidissement était de l'air filtré sur coton.

Faut-il rappeler que c'est à la suite de cet insuccès que PASTEUr 
imagina de chauffer le lait à une température plus élevée et voisine de $110^{\circ}$ a fin d'en assurer la conservation. Pour y parvenir, il augmenta la pression à l'intérieur du ballon en plongeant le col effilé de celui-ci dans du mercure. C'était l'invention de la stérilisation, et le lait a sans doute été le premier liquide stérilisé.

$$
*^{*} *
$$

En réalité, dans les conditions de la pratique, la catalase microbienne est décèlable après un temps plus ou moins long, dans tous les laits même normalement pasteurisés et conservés ensuite à l'abri de l'air.

Cette constatation ne semble pas évidemment être à l'honneur de la propreté de ces laits, mais elle doit être retenue et expliquée.

Voici en effet l'exposé de toute une série d'expériences récentes que j'ai effectuées non pas sur des laits pasteurisés, mais sur place sur des laits crus parvenant à un dépôt de laiterie d'une région Nord-Ouest, au cours des mois février-mars.

Les laits expérimentés provenaient de trente origines différentes. La traite du soir et celle du matin étaient mélangées. Ces laits se présentaient normaux, quant à leur aspect et à leur acidité. La température extérieure était d'environ $7^{\circ}$, dans la matinée où ont été effectués les prélèvements. Les conditions atmosphériques, n'étaient done pas favorables à un développement microbien, tout au moins lactique.

Les prélèvements ont été pratiqués sur chaque pot aussi aseptiquement que possible à l'aide d'une pipette plongée au centre de la masse Voici la technique suivie:

Des tubes de bouillon de viande peptoné stérile sont ensemencés avec deux gouttes de lait, puis soumis aussitôt à l'ébullition.

Des tubes vides stérilisés au préalable, sont partiellement remplis de $10 \mathrm{ec}$. de lait, de même que pour les tubes de bouillon le lait est immédiatement soumis à l'ébullition dans le tube bouché au coton.

Enfin un prélèvement de 2 ec. est effectué dans d'autres tubes pour essai à l'alizarol.

Les tubes soumis à l'ébullition, bouillon ou lait, ne sont pas brusquement refroidis, mais abandonnés au refroidissement lent, et à la température ambiante. Une durée de trois heures environ a précédé la mise à l'étuve à $37^{\circ}$ des tubes de bouillon.

En ce qui concerne ces derniers, j'ai observé pour certains, cinq heures après le prélèvement, l'apparition d'un très léger voile en surface. Sept heures après le prélèvement il était facile de classer ces tubes par degré d'importance de ce voile, celui-ci étant très manifeste dans les premiers et encore absent dans les derniers. Enfin, vingt heures après le prélèvement, tous les tubes présentaient à leur surface un voile plus ou moins épais.

Quant aux laits, ceux-ci ont été laissés à la température du 
laboratoire, voisine en moyenne de $12^{\circ}$, afin de pouvoir en suivre plus aisément les modifications. Des essais antérieurs dans lesquels la température de $37^{\circ}$ avait été utilisée ne m'avaient donné aucun renseignement, l'altération ayant été trop rapide.

Au bout de 24 heures les laits correspondant au voile maximum étaient caillés, les autrés ont suivi très exactement dans l'ordre d'importance du voile. Pour les laits ayant accusé le moindre voile, la conservation a dépassé cinq jours.

Tous ces laits ont été soumis à l'essai par l'alizarol. Bien qu'ils aient tous un degré d'acidité sensiblement identique par titrage à la soude, ils ont réagi à l'alizarol dans presque toute la gamme des teintes, depuis celle attribuée à des laits normaux, rouge-lilas jusqu'à celles attribuées à des laits acides, c'est-à-dire brun et brun-jaune.

Dans tous mes essais, il y a eu concordance absolue entre les trois déterminations effectuées, e'est-à-dire que le lait, qui ensemencé sur bouillon, a donné le voile minimum et a été le plus long à s'altérer, avait réagi en présence de l'alizarol en donnant la teinte correspondant à un bon lait, Dans le sens inverse, la même concordance s'est établie nettement, en passant par les intermédiaires dans le même ordre.

Ces trente laits, ainsi que je l'ai mentionné, étaient d'origines différentes et inconnues. A titre de témoin, en quelque sorte, j'ai examiné des laits proprement recueillis, et d'origine connue, ils ont donné lieu, ensemencés sur bouillon bouillant, à la formation d'un voile après $20 \mathrm{~h}$. environ correspondant aux meilleurs laits d'origine inconnue.

Par la suite, le caillé observé dans les tubes de lait s'est peu à peu désagrégé puis solubilisé en presque totalité, la matière grasse s'étant rassemblée au-dessus d'un liquide séreux, parfois très limpide. Ce liquide sous-jacent décomposait avec intensité l'eau oxygénée, perdait cette propriété à la suite de son chauffage à $80^{\circ}$ et avait une acidité variable d'un tube à l'autre.

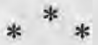

Une semblable altération des tubes de lait expérimentés sous l'influence de germes résistant à l'ébullition dont on avait pu déterminer la présence par ensemencement sur bouillon, n'est pas pour surprendre.

Dans les laits crus, en contact avec beaucoup d'autres variétés microbiennes, agissent-ils aussi profondément sur la matière azotée, ou ne sont-ils pas eux-mêmes atténués ou au contraire des activants pour ces autres espèces?

La caractéristique du voile sur bouillon, la morphologie des formes, la résistance à la chaleur indiquent le subtilis, ou une variété de subtilis, mais, cette même résistance à la chaleur et la digestion rapide du coagulum ainsi qu'une acidification du milieu lacté indique également la présence d'une variété de tyrothrix et vraisemblablement de $B$. tenuis 
attaquant le lactose, variété que WiNCKLER a obtenu des cultures de DUCLAUX.

L'apport de ces germes, ou de l'un de ces germes dans le lait se fait par l'intermédiaire de l'air de l'étable chargé de poussières de fourrages ou par les matières fécales de l'animal qui viennent souiller la traite malproprement effectuée. En temps de stabulation, on ne peut donc à priori retenir comme pollués uniquement de matières fécales, les laits à subtilis.

$$
\text { *** }
$$

Mes essais indiquent nettement que les laits examinés avaient été primitivement ensemencés plus ou moins profondément par les germes subtilis ou tyrothrix ou par les uns et les autres, et qu'ils avaient été également le siège de leur développement plus ou moins intense, développement qui a eu pour conséquence l'altération de la caséine. Celle-ci a été mise en évidence d'une manière intéressante par l'essai à l'alizarol, sans que le titrage volumétrique de l'acidité ou la dégustation aient pu la déceler.

Voici d'ailleurs les résultats comparatifs obtenus sur 24 autres prélèvements de laits crus, par l'essai à l'alizarol et le titrage de l'acidité :

\begin{tabular}{|c|c|c|c|}
\hline Teinte, & $\begin{array}{l}\text { Acidité } \\
\text { en degrés Dornic. }\end{array}$ & Teinte. & $\begin{array}{l}\text { Aciditié } \\
\text { en degrés Dornic. }\end{array}$ \\
\hline 1 & 18 & 3 & \multirow{6}{*}{$\begin{array}{c}\text { Moyenne } \\
20\end{array}$} \\
\hline 1 & \multirow{4}{*}{$\begin{array}{c}\text { Moyenne } \\
18\end{array}$} & 3 & \\
\hline 1 & & 3 & \\
\hline 1 & & 3 & \\
\hline 1 & & 3 & \\
\hline & & 3 & \\
\hline 2 & \multirow{8}{*}{$\begin{array}{c}\text { Moyenne } \\
21,1\end{array}$} & 3 & \multirow{5}{*}{$\begin{array}{c}\text { Moyenne } \\
23\end{array}$} \\
\hline 2 & & & \\
\hline 2 & & 4 & \\
\hline 2 & & 4 & \\
\hline 2 & & & \\
\hline 2 & & 5 & 25 \\
\hline 2 & & & \\
\hline 2 & & 7 & 31 \\
\hline
\end{tabular}

De l'examen du tableau ci-dessus on voit que, pour les moyennes extrêmes, les résultats obtenus avec l'alizarol ou par le titrage coïncident dans l'appréciation de la qualité du lait, mais on remarquera également que dans les teintes 1 et 3 figurent réciproquement des laits d'acidité normale et de forte acidité. Or, au point de vue de leur qualité stricte et de leur conservation ultérieure, tous les laits correspondant à la teinte 3 et au-dessus doivent être éliminés, tandis que d'autres d'une acidité DoRnIC plus élevée pourraient être à la rigueur conservés. 
J'ai fait à dessein figurer dans ce tableau des laits acides, forcément éliminés quel que soit le mode de contrôle employé.

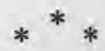

Le subtilis est-il favorable ou non au développement d'autres germes dans le lait cru? En 1897, МютснгікоFF avait démontré que des organismes du genre subtilis jouissaient du pouvoir de détruire certaines toxines. Plus tard, M. NICOLLE précise cette action antitoxique ; mais nous savons également que le subtilis, très avide d'oxygène a été utilisé dans une technique de culture des anaérobies.

D'autre part Bouska a, par une suite d'expériences nombreuses, démontré que dans une culture mixte de subtilis et de ferments lactiques, ceux-ci prenaient peu à peu le dessus après s'être développés simultanément avec rapidité. Il est incontestable comme je l'ai démontré que des laits crus ou pasteurisés qui ont subi l'action des peptolysants, seront un meilleur terrain de culture pour d'autres germes susceptibles de s'y développer ultérieurement, du fait des matériaux peptonisés, plus directement assimilables qu'ils y rencontreront.

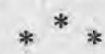

Dans la pratique, indépendamment du gouttage, qui n'est pas un procédé scientifique, qui n'est pas recommandable au point de vue de l'hygiène, mais qui n'en est pas moins assez précis lorsqu'il est pratiqué par une personne exercée, on se borne généralement pour apprécier un lait à déterminer l'altération, du lactose par titrage volumétrique de l'acidité, en tenant compte d'une acidité naturelle exprimée en acide lactique.

L'altération de la matière albuminoïde devrait être également déterminée, car elle peut jouer un rôle important dans certains cas.

En laiterie, on observe fréquemment des laits épais, qui " attachent » dans le pasteurisateur, qui plus tard, chez le consommateur supporteront mal l'ébullition et feront l'insuccès de certaines préparations culinaires sans pour cela être acides. De tels laits ont été apparemment le siège d'une coagulation partielle sous l'influence d'une diastase microbienne, laquelle détruite par la température de pasteurisation, n'en a pas moins modifié antérieurement la texture de la caséine, done modification qui subsiste dans le lait après son traitement.

Dans l'industrie très spéciale du lait stérilisé, la présence de ces germes thermo-résistants et coagulants est encore plus néfaste, puisque c'est parfois très longtemps après la stérilisation, alors que le lait est en magasin ou expédié au loin, que leur action se produit, causant ainsi une perte grave ou une dépréciation de la marque.

En fromagerie, il est hors de doute que l'action de ces mêmes germes vient modifier d'une manière insoupçonnée, parleurs sécrétions diastasi- 
ques coagulantes, l'action de la présure elle-même, et contrarier probablement la suite prévue des opérations, à moins qu'elle ne le favorise.

Faut-il voir une simple coïncidence ou une action réelle de ces germes dans deux cas qui se présentent dans la fabrication du gruyère? Le premier c'est cette prédilection des vieux fromagers pour l'emploi d'une macération prolongée des caillettes effectuée par leurs soins et en surface de laquelle on observe un voile caractéristique.

Le second est cette pratique fréquemment utilisée qui consiste à adjoindre au lait frais en cuve une quantité importante de lait "mûri » dans des rondeaux refroidis.

Au point de vue de la maturation de la crème destinée au barattage, il est indéniable que ces mêmes germes viennent jouer un rôle important et néfaste contrariant ou devançant celui des ferments lactiques réels dont cette même crème se trouve parfois insuffisamment ensemencée.

Enfin dans la préparation de la caséine lactique, telle qu'elle s'effectue dans les dépôts de laiterie, j'ai à plusieurs reprises constaté des «accidents ") de fabrication se traduisant par des caillages anormaux ou des pertes en rendement importantes. II y avait faible acidification, elle n'était pas le fait des ferments lactiques, mais de germes acidifiants peptonisants et solubilisants.

A certaines périodes de l'année, il semble qu'il y a prédominance dans le lait de germes thermo-résistants sur les ferments lactiques vrais, les premiers peuvent évoluer à une température plus basse que les seconds, ils sont susceptibles à eux seuls de provoquer le caillage ou de l'activer s'il doit s'exercer sous l'influence des ferments lactiques apportés par la suite.

Il y a lieu de faire observer que le subtitis ou les subtilis et les tyrothrix ici envisagés, ne sont pas, bien qu'agissant sur la matière azotée, des agents de putréfaction au sens strict, producteurs d'hydrogène sulfuré et d'indol. Ils ne sont pas pathogènes, nous en absorbons de grandes quantités dans les fromages, les fruits crus, les salades, etc., et, paut-être, ainsi qu'on le verra plus loin, ne sont-ils pas tout à fait incompatibles avec une hygiène rigoureuse.

Nous savons, d'autre part, que l'évolution du subtilis est rapide et fonction de la température; à $21^{\circ}$ par exemple, un bâtonnet issu d'une divison demande einq quarts d'heure pour se partager à son tour, à $35^{\circ}$,il suffit de 20 minutes. Quant aux spores, leur germination peut se faire en 12 heures à la température ordinaire. D'après BUCHNER, elle est hâtée si l'on fait bouillir au préalable les spores et qu'on laisse refroidir lentement le milieu, l'évolution se manifeste alors dans les 2 à 3 heures : ce sont les conditions de mes expériences sur bouillon.

On serait tenté de rapprocher cette constatation de ce qui se passe dans la pasteurisation, mais là le refroidissement est brusque et la température subie moins élevée. 
Dáns la pratique, le lait se trouve ensemencé dès son contact à l'air non seulement de spores mais aussi de germes actifs, la température et le lent refroidissement sont très favorables au développement de ces germes peptolysants, que vient activer comme on le sait le mélange des traites.

$$
\text { ** * }
$$

L'alizarol m'a permis de préciser ces faits, son emploi en laiterie me semble des plus recommandables pour parfaire la classification des laits. Je dois signaler cependant que les diverses marques d'alizarol ne réagissent pas d'une manière identique, et qu'il importe de s'en tenir à une marque ayant fait ses preuves et dont on a l'habitude d'observer les résultats (1).

$$
\text { *** }
$$

Qu'il me soit permis de rappeler, pour terminer, bien qu'elle ne relève pas strictement de la science laitière une étude antérieurement publiée pour mettre en évidence l'action peptonisante du subtilis. Elle m'a valu à son époque une remarque d'une haute personnalité scientifique qui a attribué mes résultats à l'action des vitamines, alors peu étudiées. Ne m'étant pas spéoialisé dans cette question très complexe, il m'est impossible de discuter, s'il y en a un, le rapport entre lè subtilis et les vitamines ou une sorte de vitamine mais je rappelerai, à titre de simple coïncidence, le rôle de la balle de riz, riche en subtilis, pour combattrele béribéri, celui des fruits et salades, riches en subtilis, par définition, comme apport de vitamines, et enfin la constatation de maladies de carence par ingestion d'aliments stérilisés donc privés des thermorésistants que ne parvient pas à détruire l'ébullition, couramment utilisée pour la cuisson des aliments.

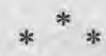

Voici l'exposé de cette étude:

Dans de précédents travaux j'ai été conduit à rappeler l'influence qu'exerçait sur un liquide riche en matières albuminoïdes tel que le lait, le développement des microbes du genre subtilis, microbes que l'on rencontre à l'état latent dans la poussière des fourrages et à la surface de la plupart des graines. L'action de ces germes transforme progressivement la matière albuminoïde en peptones et les autres germes, notamment les ferments lactiques que le lait est susceptible de collecter, trouvent de ce fait un aliment plus favorable à leur prolifération.

La pasteurisation du lait qui interrompt pour un certain temps l'action de ces agents peptonisants, puisqu'elle n'en détruit pas les spores, ne corrige pas les modifications antérieures qu'ils ont fait subir à la matière albuminoïde. Il s'ensuit que, toutes choses égales d'ailleurs,

(1) L'alizarol employé provenait de la maison Lienhart de Strasbourg, il a été utilizé en solution dans l'alcool à $65^{\circ}$. L'échelle des teintes est celle du $\mathrm{D}^{\mathrm{r}}$ Morres. 
le lait se maintiendra d'autant mieux après pasteurisation qu'il aura été conservé aussitôt après la traite, à l'abri des poussières des étables.

On était en droit de se demander si cette action, néfaste dans le cas du lait, de ces germes peptonisants, ne pouvait être utilisée avec profit dans le cas de la nourriture des animaux en donnant préeisément à ceuxci des aliments dont la matière azotée, si faible soit-elle, ait été partiellement peptonisée avant l'ingestion.

Les expériences que j'ai exécutées pour la vérification de ce fait ont porté sur 50 souris divisées en deux lots : témoins et essais. Ces animaux ont été nourris pendant quinze jours de la façon suivante, les conditions d'humidification étant strictement identiques:

Lẹs 25 souris témoins reçoivent des graines d'avoine aplaties et stérilisées en milieu humide à $120^{\circ}$.

Les 25 souris essais reçoivent les mêmes graines mais arrosées au préalable avec une dilution aqueuse tenant en suspension des fragments dissociés de voile de $B$. subtilis provenant d'une culture sur bouillon et débarrassés par lavages successifs de toutes traces sensibles de matière azotée.

Voici les résultats obtenus :

\section{POIDS GLOBAL DES SOURIS.}

\begin{tabular}{cccc}
\multicolumn{2}{c}{ Avant l'expérience. } & \multicolumn{2}{c}{ Après l'expérience. } \\
Témoins: & Essais. & Témoins. & Essais. \\
268 & 266 & 296 & 315
\end{tabular}

Augmentation des témoins p. 100 du poids vif ...... 9.4

Augmentation des essais ................... 15.5

Les souris ont continué à engraisser dans les deux cas, mais avec une très sensible différence en faveur des essais, qui met ainsi nettement en évidence l'influence du subtilis.

Les graines données aux animaux, toutes les douze heures environ, restent à leur disposition d'une distribution à l'autre, sans aucune précaution aseptique spéciale, elles se contaminent done progressivement au contact de l'air et des déjections et perdent leur caractère de stérilité. Les souris témoins ne se trouvent done pas dans un état rigoureux de carence, et n'ont nullement souffert de leur traitement. On serait cependant tenté de rapprocher ces expériences de celles exécutées notamment par PoRTier pour mettre en évidence le rôle des organismes qu'il a dénommés "symbiotes". Le but de mes essais n'est pas de confirmer la nécessité pour l'entretien de la vie, de la présence d'un " microorganisme doué de propriétés biochimiques remarquables et qui a tous les caractères du B. subtilis " (Marchoux, Annales de l'Inst. Pasteur, t. XVII, p. 177, loc. cit.) mais de démontrer que le B. subtilis 
en addition préalable à des graines humidifiées agit sur la matière azotée qu'il peptonise et la rend ainsi mieux utilisable pourl'alimentation.

\title{
BIBLIOGRAPHIE
}

O. Jensen. Revue générale du lait 1906.

J. Sarthou. C. R. Ac. Se. t. CL. 1910 p. 119.

J. SARthou. Journal de Pharmacie et de Chimie 1910.

M. Nicolle. Ann. Inst. Pasteur, t. XXI, 1907.

Bouska. Revue générale du lait, t. III, 1903.

Macḱ. Traité de Bactériologie, t. II, 1913.

Portier. C. R. t, A. Sc. CLXV, p. 267.

Marohoux. Ann. Inst. Pasteur, t. XVII, p. 177, loc. cit.

M. Fouassier, C. R. Ac. Sc. t. CLXX, 1920, p. 145.

M. FouAssier. $C, R$. Ac. Sc. t. CLXXI, 1920, p. 327.

M. Fovassier. C. R. Académie d'Agriculture, t. VII, 1921, p. 706.

\section{EXPÉRIENCES TECHNIQUES CONCERNANT L'INFLUENCE DE LA TEMPÉRATURE DU BARATTAGE SUR LA TENEUR EN MATIËRE GRASSE DU BABEURRE, PROVENANT DE CRÈME RÉFRIGÉRÉE A TRÈs BASSE TEMPÉRATURE.}

\author{
par W. Van DAM.
}

Par l'élévation de la température du barattage, la teneur en matière grasse du babeurre, provenant d'une crème déterminée et travaillée, pour le reste, dans les mêmes conditions, augmente. Ce fait est universellement connu, et fut constaté dans nombre d'expériences. Encore dernièrement au Danemark (1), des expériences sur très grande échelle en donnèrent confirmation : 24 expériences furent faites dans deux fabriques; il y eut trois températures différentes, dont l'écart était de $+3^{\circ} \mathrm{C}$. ; les moyennes suivantes furent établies pour la teneur en matière grasse du babeurre : Température la plus élevée : $0,877 \%$. Température intermédiaire : $0,586 \%$. Température la moins élevée : $0,490 \%$.

Pour autant qu'il m'est connu, on n'utilisa jusqu'ici dans de telles expériences techniques que de la matière grasse de crème, qui n'était pas dans des conditions d'équilibre à la suite de réfrigération à trè̀ basse température, et comme il fut constaté dans les expériences de laboratoire que dans le babeurre provenant de crème dont la matière grasse n'est pas dans des conditions d'équilibre, la teneur en matière grasse est toujours plus élevée qủe dans celui de crème où ces conditions ont été réalisées (2), il était intéressant d'examiner, à ce sujet, les

(1) 120 de Beretning fra Forsogslaboratoriet, 1926

(2) Le Lait, 1928, p. 369. 